\title{
Influence of polydispersity on the microstructure of frictionless disc packings under simple shear
}

\author{
Nicolas Estrada ${ }^{1, \star}$ and William-Fernando Oquendo ${ }^{2, \star \star}$ \\ ${ }^{1}$ Departamento de Ingeniería Civil y Ambiental, Universidad de los Andes, Bogotá, Colombia \\ ${ }^{2}$ Departamento de Ingeniería Industrial, Universidad de la Sabana, Chía, Colombia
}

\begin{abstract}
By means of 2D Contact Dynamics simulations, we explored the effects of the shape and size span of the grain size distribution (GSD) on the microstructure of a sheared granular packing, in terms of its packing fraction and the connectivity of its contact network. We focused on GSDs that can be represented by a power law, which are widely used in several engineering and industrial contexts. The interest in power law GSDs originates in the works of Fuller and Thompson in 1907 and 1919, in which it was found that a maximum density is obtained for a power law GSD with an exponent of 0.5. This disagrees with recent DEM results where the densest packing is obtained for linear cumulative volume distributions. In order to explore this discrepancy, we performed systematic simulations in which we varied both the size span (the ratio between the largest and the smallest diameter) and the exponent (shape) of the distribution. We find that the exponent equal to 0.5 produces the highest density for all size spans. Furthermore, the proportion of rattlers and coordination were also analyzed, showing that the system's connectivity is strongly affected by both the size span and the shape of the distribution.
\end{abstract}

\section{Introduction}

It is well known that the grain size distribution (or polydispersity) plays an important role on the dynamics of granular materials. For instance, polydispersity strongly affects the material's packing fraction, which in turn affects its mechanical response. Therefore, it is of paramount importance to choose the appropriate grain size distribution (GSD) when the material's design targets some specific property, as it is done for the granular phase of composite materials such as Portland and asphalt concrete, or in the granular bases in roadways pavements.

The relationship between polydispersity and packing fraction has been studied for more than a century. The first experimental works are probably those of Fuller and Thompson in 1907 [1] and Taylor in 1919 [2]. Fuller and Thompson found that the optimal GSD (i.e., that with the highest density) is the one for which the cumulative volume distribution is described by

$$
\rho=\left(d / d_{\max }\right)^{0.5},
$$

where $\rho$ is the mass percentage of particles with diameter smaller than $d$, and $d_{\max }$ is the maximum diameter of the sample. In the years that followed, several researchers explored different materials, used in different industrial contexts, finding smaller values for the optimal exponent. In particular, the optimal exponent decreased as the amount of fines in the samples increased [3-6]. A useful extension,

^e-mail: n.estrada22@uniandes.edu.co

$\star \star$ e-mail: william.oquendo@unisabana.edu.co which includes a minimum diameter $d_{\min }$ was proposed in [7] and reads

$$
\rho=\left(\frac{d-d_{\min }}{d_{\max }-d_{\min }}\right)^{\eta},
$$

in which $d_{\min }$ and $d_{\max }$ control the size span of the GSD, while $\eta$ controls its shape. For example, $\eta=0$ corresponds to a mono-sized material.

Numerous theoretical studies have also been conducted in order to develop analytical expressions that allow for predicting the packing fraction, given a certain GSD. However, these expressions only give good predictions for discrete GSDs (binary or ternary mixtures) [817].

Another possible approach is to build and analyze arrangements of particles according to a geometrical rule, as those presented in [13, 18-25]. These models represent a tool of great value since they allow for exploring a wide range of systems, configurations and size distributions, which is something that cannot be easily done experimentally or theoretically. In particular, in [23] a large set of GSDs were built and compared by using the cumulative $\beta$-distribution, finding that the GSD that produces the densest packing is the uniform distribution by volume fractions (i.e., $\eta=1$ in eq. (2)). This disagrees with the optimal exponent of $\eta=0.5$ proposed in [1, 2], although these experiments were based on mixtures of fine and coarse grains, and the model in [23] is two dimensional.

During the past decades, several works have used the Discrete Element Method (DEM) to study the effects of 


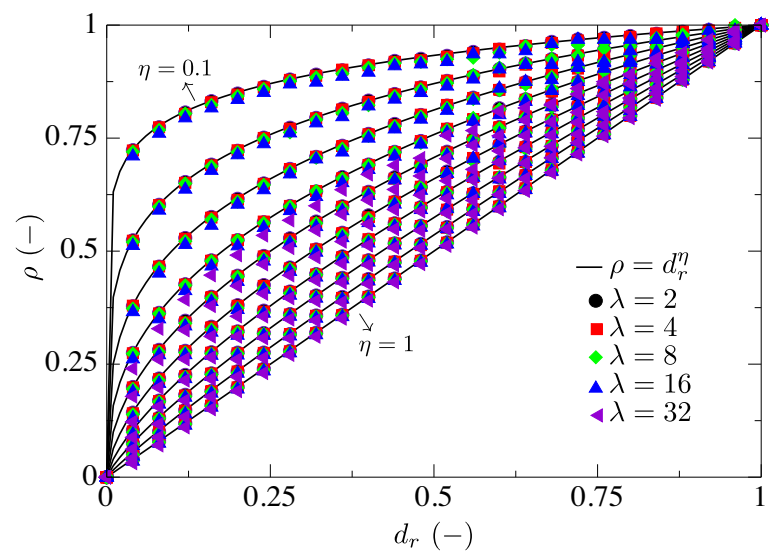

Figure 1. (Color online) Grain size distributions for all samples. The black line corresponds to eq. (2)

the GSD on different properties of granular packings [2631]. In this paper, we use DEM to explore the influence of both the size span and the shape of the GSD on the packing fraction and connectivity, for two-dimensional samples of frictionless discs under simple shear.

\section{Numerical procedure}

The numerical samples were composed of approximately 10000 discs, with a GSD that followed a power law such as that presented in Eq. 2. The size span of the GSD was described by the size ratio $\lambda=d_{\max } / d_{\min }$, and the shape of the GSD was controlled by the exponent $\eta$. Systems were built for the following set of parameters: $\lambda \in(2,4,8,16,32)$ and $\eta \in(0.1,0.2, \ldots, 1)$. For this purpose, the range of diameters $\left(d_{\min }, d_{\max }\right)$ was divided into ten subranges, and, inside these subranges, a uniform distribution by number of grains was used.

Figure 1 shows the GSDs (i.e., the cumulate area distributions) of all samples; $\rho$ is the area percentage of particles with a reduced diameter smaller than $d_{r}=(d-$ $\left.d_{\min }\right) /\left(d_{\max }-d_{\min }\right)$. It can be seen that the built GSDs closely follow the theoretical curves. For $\lambda=32$, only samples with $\eta \geqslant 0.4$ were built, since it was seen that smaller values of $\eta$ would require a larger number of grains in order to obtain a good fit (i.e., a maximal vertical offset of 0.03 ) with the theoretical curve.

All samples were periodic on the horizontal direction. These samples were first compressed under a constant stress $\sigma_{c}$, and then the systems were sheared horizontally with shear speed $v_{s}$ under the same confining stress. The inertial number $I$ was defined as

$$
I=\frac{\dot{\gamma}\langle d\rangle}{\sqrt{\sigma_{c} / \varrho}},
$$

where $\dot{\gamma}$ is the shear rate and $\varrho$ is the grains' density. In all shear tests $I \simeq 10^{-4}$, which means that these tests can reasonably be considered as quasi-static [32].
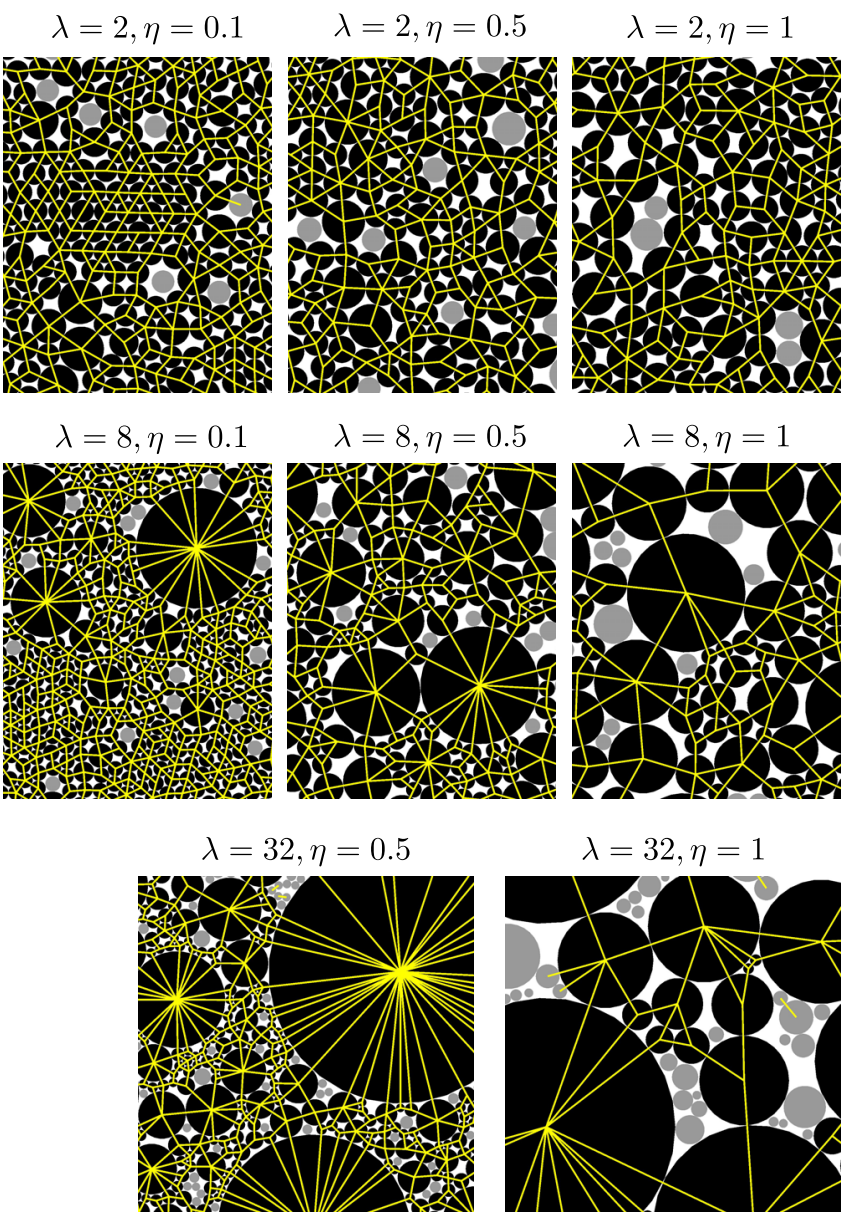

Figure 2. (Color online) Contact network for $\eta=0.1,0.5,1.0$ and $\lambda=2,8,32$. Yellow (white) lines join the center of particles in contact. The length scale is the same on all snapshots.

\section{Results}

\subsection{Contact network}

Figure 2 shows the contact network for eight systems with different values of the size span $\lambda$ and exponent $\eta$ of the GSD. For $\eta=0.1$ a big part of the system is almost monodisperse and spontaneous crystallization occurs to some degree. Large size spans are characterized by a disordered structure but also by a higher proportion of rattlers (i.e., grains that do not participate in the force-carrying backbone). For $\eta=0.5$ it seems that the amount of smaller particles is appropriate to fill better the voids between the larger particles. Simultaneously, the contact network's connectivity is also enhanced.

\subsection{Packing density}

The packing density was quantified by means of the packing fraction, defined as

$$
v=\frac{A_{g}}{A},
$$

where $A_{g}$ is the area covered by the grains, and $A$ is the total area of the sample. Figure 3 shows $v$ as a function of 


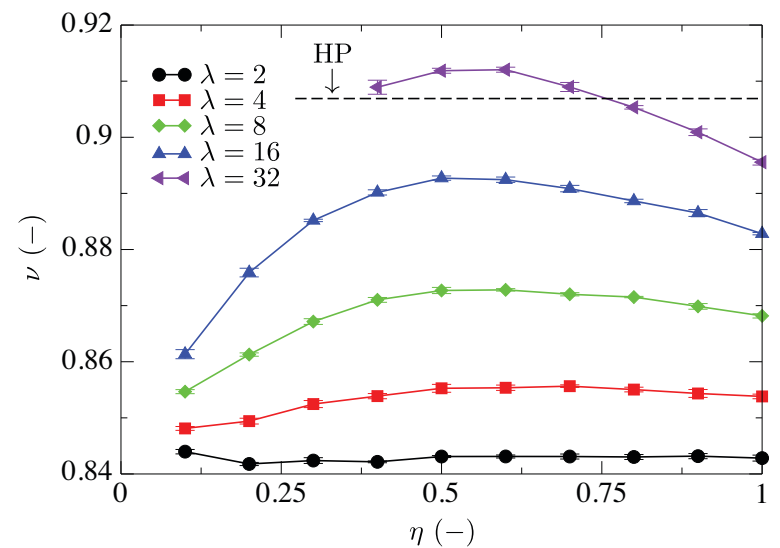

Figure 3. (Color online) Packing fraction $v$ as a function of $\eta$ for all values of $\lambda$. Error bars indicate the standard deviation from several measures at the steady state. The dashed line indicates the packing fraction of a set of mono-disperse discs packed on a hexagonal arrangement $-\mathrm{HP}\left(\nu_{\mathrm{HP}}=\pi / 2 \sqrt{3} \simeq 0.9069\right)$

$\eta$ for all values of $\lambda$. First, it can be seen that $v$ increases with $\lambda$, as shown also in $[28,31]$, as small particles fill the pores between the larger particles. Second, it can be seen that $v$ varies non-monotonically with $\eta$, showing a maximum around $\eta \simeq 0.5$. Therefore, the maximum density is obtained when the GSD follows a power law with an exponent close to 0.5, as suggested by Fuller and Thompson $[1,2]$, and not for the uniform distribution by volume fractions, as suggested by Voivret [23]. This discrepancy could be due to the fact that the set of GSDs explored in [23] did not include a power law of exponent 0.5. In addition, the samples analyzed in [23] were built geometrically, while the systems analyzed in this paper were built mechanically. Third, it is also shown that $v$ can exceed the packing fraction of system of mono-disperse discs in hexagonal packing (i.e., $v=\pi / 2 \sqrt{3} \simeq 0.9069$ ).

\subsection{Connectivity}

Figure 4 shows the proportion $\kappa$ of rattlers as a function of $\eta$ for all values of $\lambda$. It can be seen that $\kappa$ increases with both $\lambda$ and $\eta$. For $\lambda=32$ and $\eta=1$, almost $45 \%$ the particles are rattlers, which means that the force-carrying backbone is only composed by approximately half of the particles. Figure 5 shows the average coordination number $\left\langle n_{c}\right\rangle$ as a function of $\eta$ for all values of $\lambda$. It can be seen that $\left\langle n_{c}\right\rangle$ decreases with both $\lambda$ and $\eta$, as a result of the increasing number of rattlers. It is also shown $\left\langle n_{c}\right\rangle$ for the force-carrying backbone, which is very close to 4 , as is expected for a two-dimensional system of frictionless discs. It is interesting to note that systems with $\eta=0.5$ are notably better connected than those with $\eta=1$, which should have an important effect in macroscopic mechanical properties such as Coulomb cohesion and Stiffness.

\section{Conclusions and perspectives}

In this paper we explored the effects of the shape and size span of the grain size distribution (GSD) on the mi-

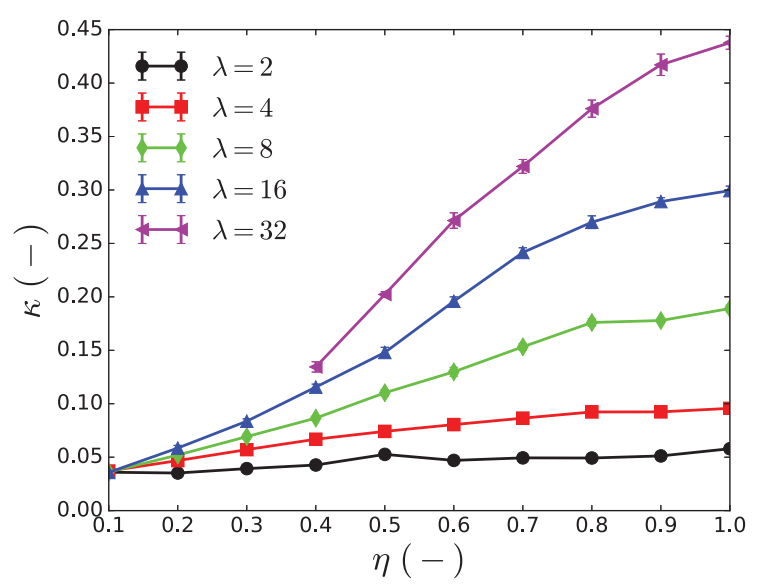

Figure 4. Proportion $\kappa$ of rattlers as a function of $\eta$ for all values of $\lambda$. Error bars indicate the standard deviation from several measures at the steady state.

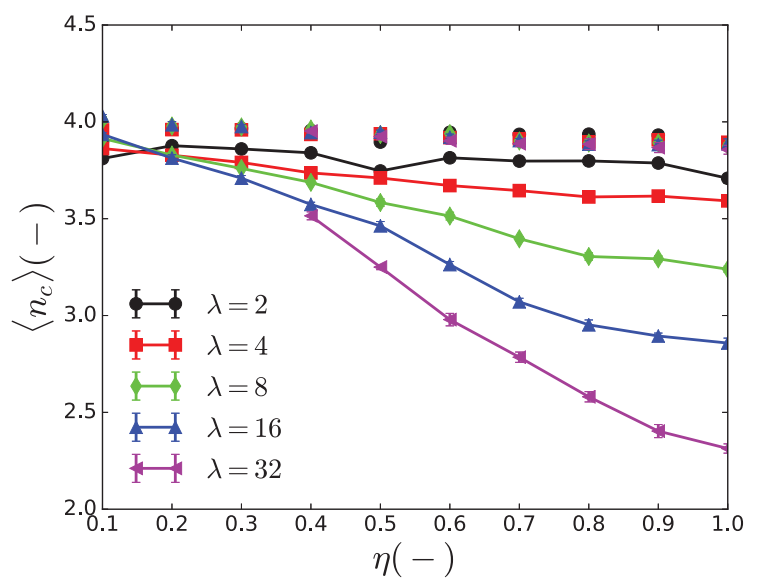

Figure 5. Coordination number $\left\langle n_{c}\right\rangle$ including all particles (lines and points) and for the force-carrying backbone only (only points). Error bars indicate the standard deviation from several measures at the steady state.

crostructure of a sheared granular packing. This microstructure was described in terms of the packing fraction and the connectivity of the contact network.

We showed that the GSD that produces the densest packing corresponds to a power law with an exponent close to $\eta=0.5$ (see equation (2)), in agreement with the results of Fuller and Thompson [1, 2] for 3D experiments. Our results enhance and extend the validity of the Fuller and Thompson optimal GSD [1,2] to 2D systems of frictionless discs. Qualitatively it was suggested that the high packing fraction obtained with this distribution is due to a higher proportion of small particles that allows for efficiently filling the voids between the larger particles, an effect which increases with increasing size span.

Or results disagree with those presented in [23], where the densest packing was found to be the the uniform distribution by volume fractions (i.e., a power law GSD with $\eta=1)$. It is important to note that the set of GSDs ex- 
plored in [23] did not include a power law of exponent 0.5 . Moreover, the samples analyzed in [23] were built geometrically, while the systems analyzed in this paper were built mechanically.

Finally, we showed that both the size span and shape of the GSD increase the proportion of rattlers and decrease the coordination of the force-carrying backbone. It is also shown that the distributions with $\eta=0.5$ are not only denser than those with $\eta=1$, but also better connected. This is important because it could have a major effect on macroscopic mechanical properties such as Coulomb cohesion and stiffness.

This work can be extended by exploring the role of the size span and shape of the GSD in a three dimensional system. Furthermore, we are currently exploring the links between the GSD and other macroscopic properties beyond shear strength. This should be useful to better understand the effects of polydispersity and use this knowledge for industrial applications in which the GSD can, or even needs to be, controlled.

\section{Acknowledgements}

The authors would like to thank J. P. Carmona and J. M. Torres for their help with numerical simulations.

\section{References}

[1] W.B. Fuller, S.E. Thompson, Transactions of the American Society of Civil Engineers LIX, 1 (1907)

[2] F.W. Taylor, S.E. Thompson, A treatise on concrete, plain and reinforced (John Wiley \& Sons, 1919)

[3] A.N. Talbot, F.E. Richart, Illinois Univ Eng Exp Sta Bulletin (1923)

[4] J.M.S. Shilstone, Concrete International: Design and Construction 12, 33 (1990)

[5] P.N. quiroga, D.W. Fowler, Tech. Rep. 104-2, International Center for Aggregates Research, Austin, TX (1994)

[6] H.J.H. Brouwers, H.J. Radix, Cement and Concrete Research 35, 2116 (2005)

[7] J.E. Funk, D.R. Dinger, Predictive process control of crowded particulate suspension (Springer US, 1994)

[8] C.C. Furnas, Tech. Rep. 2894, Department of Commerce, Bureau of Mines (1929)

[9] R.F. Fedors, R.F. Landel, Powder Technology 23, 225 (1979)
[10] N. Ouchiyama, T. Tanaka, Industrial \& Engineering Chemistry 23, 490 (1984)

[11] T. Stovall, F. de Larrard, M. Buil, Powder Technology 48, 1 (1986)

[12] A.B. Yu, N. Standish, Powder Technology 55, 171 (1988)

[13] T. Aste, Physical Review E 53, 2571 (1996)

[14] H. Brouwers, Physical Review E 74, 031309 (2006)

[15] H. Brouwers, Physical Review E 84, 042301 (2011)

[16] H. Brouwers, Physical Review E 87, 032202 (2013)

[17] H.J.H. Brouwers, Physical Review E 89, 052211 (2014)

[18] A. Bezrukov, D. Stoyan, M. Bargiel, Image Anal. Stereol 20, 203 (2001)

[19] M. Wackenhut, H. Herrmann, Physical Review E 68, 041303 (2003)

[20] H.J. Herrmann, R. Mahmoodi Baram, M. Wackenhut, Physica A: Statistical Mechanics and its Applications 330, 77 (2003)

[21] K. Sobolev, A. Amirjanov, Advanced Powder Technology 15, 365 (2004)

[22] K. Sobolev, A. Amirjanov, Powder Technology 141, 155 (2004)

[23] C. Voivret, F. Radjaï, J.Y. Delenne, M. El Youssoufi, Physical Review E 76, 021301 (2007)

[24] J.F. Jerier, V. Richefeu, D. Imbault, F.V. Donzé, Computer Methods in Applied Mechanics and Engineering 199, 1668 (2010)

[25] S.D.S. Reis, N.A.M. Araújo, J.S. Andrade, jr., H.J. Herrmann, EPL (Europhysics Letters) 97, 18004 (2012)

[26] P. Stroeven, M. Stroeven, Cement and Concrete Research 29, 1201 (1999)

[27] M. Wackenhut, S. McNamara, H. Herrmann, The European Physical Journal E 17, 237 (2005)

[28] C. Voivret, F. Radjaï, J.Y. Delenne, M. El Youssoufi, Physical Review Letters 102, 178001 (2009)

[29] B. Yohannes, K.M. Hill, Chaos: An Interdisciplinary Journal of Nonlinear Science 82, 061301 (2010)

[30] D.H. Nguyen, E. Azéma, F. Radjaï, P. Sornay, Physical Review E 90, 012202 (2014)

[31] D.H. Nguyen, E. Azéma, P. Sornay, F. Radjaï, Physical Review E 91, 032203 (2015)

[32] GDR-MiDi, Eur. Phys. J. E 14, 341 (2004) 